\title{
Editorial
}

\section{Workshop Mujer, Óptica y Fotónica}

Hola a todas y todos,

El bajo número de mujeres en ciertos campos de la Óptica y la Fotónica, como en general en los campos de la Física y la Ingeniería, es un hecho ampliamente probado. Solo un 28 \% del alumnado universitario que estudia carreras STEM (acrónimo inglés que aglutina ciencia, tecnología, ingeniería y matemáticas) en España son mujeres, y teniendo en cuenta que, según la Comisión Europea para 2020 se necesitarán un millón de perfiles tecnológicos, esta brecha de género, que comienza ya en etapas muy tempranas, será aún más profunda en relación con las oportunidades laborales que puedan tener las mujeres en las futuras profesiones en estos campos. Lo mismo ocurre durante la carrera investigadora, en la que el número de científicas desciende significativamente en la etapa pre- y post-doctoral, llegando en muchos casos a porcentajes ridículos en las escalas superiores de dicha carrera (Profesora/Catedrática de Universidad, PI de Grupo de Investigación), así como en presidencias de sesión en congresos o en premios científicos.

A las diferentes políticas puestas en marcha por los Gobiernos y la Comisión Europeos, para reducir esta brecha de género en Ciencia, se unen las iniciativas de las diferentes sociedades científicas internacionales del área de la Óptica y la Fotónica (OSA, SPIE), a las que recientemente se ha unido la Sociedad Española de Óptica (SEDOPTICA). Durante la última Reunión Nacional de Óptica (RNO2018, Castellón) se aprobó con amplio apoyo por parte de los miembros de SEDOPTICA la creación del Área de Mujeres, Óptica y Fotónica (SEDOPTICA-MOF) de SEDOPTICA para contribuir a la reducción de la brecha de género en el campo de la Óptica y la Fotónica.

Menos de un año después de su creación, SEDOPTICA-MOF, ha organizado el I Workshop de Mujeres, Óptica y Fotónica @SEDOPTICA (6 Septiembre, Madrid, Campus Central CSIC) con el objetivo de fortalecer la ya existente red de mujeres en Óptica y Fotónica en diferentes entornos (universitario, científico, empresarial). Para ello reunimos a un amplio número de ponentes de diferentes ámbitos docentes, universitarios, científicos y empresariales para tener una visión global de la situación de la mujer en el mundo de la Óptica y la Fotónica en España. El evento ha logrado reunir a más de 120 estudiantes, científicas, profesoras, profesionales del área y emprendedoras, de todos los puntos geográficos, conformando una foto de grupo en el que han quedado representadas varias generaciones de científicas españolas. Como expresaron varias de las asistentes, la jornada se vivió como un acontecimiento histórico que puede ser el catalizador de diferentes iniciativas para reducir los problemas de género en Óptica y Fotónica.

La jornada comenzó con la apertura institucional, llevada a cabo por Elena Domínguez, Vicepresidenta de Relaciones Internacionales del CSIC; seguida por María Sagrario Millán, Vicepresidenta 
de SEDOPTICA, y por Ángeles Heras, Secretaria de Estado de Universidades, Investigación, Desarrollo e Innovación del Ciencia, Innovación y Universidades. Todas ellas resaltaron la necesidad de eventos como este Workshop para eliminar la persistente brecha de género en Ciencia.

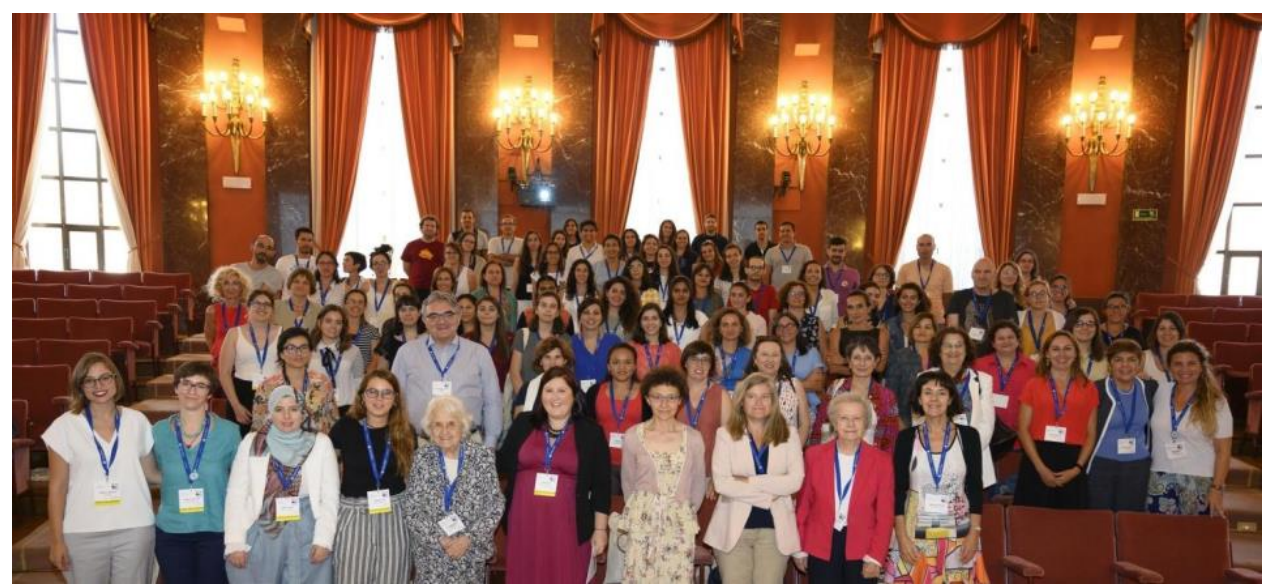

Foto de grupo del I Workshop Mujer, Óptica y Fotónica

A continuación, María Viñas (investigadora postdoctoral IO-CSIC) y Clara Benedí (investigadora predoctoral IO-CSIC), coordinadoras de SEDOPTICA-MOF, y miembros del comité organizador del evento, presentaron algunas cifras sobre la situación de la mujer en el Área de la Óptica y la Fotónica en España, así como las diferentes iniciativas que quiere desarrollar SEDOPTICA-MOF para cambiar esas cifras. Uno de los objetivos principales será la creación de informes que proporciones información sobre la verdadera situación de las mujeres en Óptica y Fotónica en España. También el desarrollo de programas de SEDOPTICA-MOF como "Conoce a las investigadoras" o el "Programa de Mentoras".

El primer evento de la jornada fue una Mesa redonda "Una visión personal sobre la Óptica \& Fotónica", moderada por Paloma Reyes (investigadora predoctoral, IO-CSIC), en la que investigadoras en diferentes estadíos de su carrera profesional (María Luisa Calvo (Catedrática de Óptica UCM), Ana Rodríguez (investigadora predoctoral UPC), Marina Díaz Michelena (INTA) y Verónica González (investigadora postdoctoral, Aix-Marseille Université)), y compartieron sus experiencias comunes y sus diferentes puntos de vista.

A continuación, las Profesoras Laura Lechuga (Instituto Catalán de Nanociencia y Nanotecnología (ICN2-CSIC)) e Inmaculada Pascual (Catedrática de Óptica en Universidad de Alicante) nos ofrecieron una visión global de sus intereses investigadores, biosensores a escala nanométrica y materiales holográficos respectivamente, y de sus propias experiencias vitales en el desarrollo de dichas carreras, con algunos consejos para las jóvenes investigadoras presentes en el evento.

Durante la pausa del café (patrocinado por la plataforma Fotónica 21) pudimos disfrutar de la exposición Exposición "Las chicas somos guerreras...y también ingenieras", resultado de la participación del MNCN en el Proyecto Europeo Hypatia (H2020 2015-18). 
Con las fuerzas repuestas, fue el turno de nuevas charlas, impartidas por las profesoras de investigación Susana Marcos (IO-CSIC), y Carmen Vázquez (UC3M), sobre Óptica Visual y Biofotónica y Optoelectrónica.

Llegó el turno de la divulgación, tan importante a la hora de recortar la brecha de género en Ciencia, animar a las estudiantes a elegir carreras científicas, y aumentar la diversidad y multiculturalidad de la comunidad científica, con una serie de presentaciones cortas a cargo de Sara Aissati (investigadora predoctoral IO-CSIC, IOSA), Aitor Villafranca (investigador postdoctoral IO-CSIC, Prisma), Carmen Martín (estudiante del Grado de Física en UVa, Physics League) y Laura Rego (investigadora predoctoral ALF-USAL, OSAL) que nos contaron las actividades de divulgación que realizan dentro de sus respectivas organizaciones de investigadores. Para cerrar la sesión de divulgación, la investigadora Elena Pinilla (Centro de Tecnología Nanofotónica de Valencia, vicepresidenta de la RSEF) nos habló de su experiencia individual como divulgadora, poniendo el foco en actividades relacionadas con mujeres y ciencia, como el $11 \mathrm{~F}$.

Durante la comida, las asistentes pudieron ver la exposición "Investigadoras en la Luz y las Tecnologías de la Luz", creada para el año internacional de la luz 2015 por la FECyT y la RSEF junto a la que hicimos la foto de las Investigadoras pre- y post-doctorales ganadoras de las bolsas de viaje del congreso: Ana Isabel Gómez (USC), Laura Caliò (CSIC-ICMSE), Sara Peña (UPC), Martina Delgado-Pinar (UV), Elena Cabello (ICMS-CSIC), Victoria Esteso (ICMS-CSIC), María Tomás (UA), Amal Zaytouny (UPC), Guadalupe Lopez (UMH), Carla Rodríguez (UAB), Cantia Belloso (Uva), Leticia Carrión (Photonicsens-UV), Ana Álvarez (Uva) y Teresa Loureiro (Universidade do Minho). Todas ellas pudieron charlar con la investigadora jubilada del IO-CSIC, Juana Bellanato, pionera de la Espectroscopía en España.

La tarde comenzó con tres exposiciones breves de mujeres provenientes del ámbito de la industria española de la Óptica y la Fotónica: Rosa María Sánchez (Secpho), Azahara Almagro (Fyla), y Nerea Otero (Aimen), que nos hablaron de las empresas en las que trabajan y de su trayectoria profesional en las mismas.

Por último la mesa redonda sobre "Sociedades científicas", moderada por Francesca Gallazzi (investigadora predoctoral IO-CSIC) en la que participaron María Josefa Yzuel (ex-presidenta Sociedad internacional Óptica y Fotónica, SPIE), Pilar López Sancho (Presidenta Comisión de Mujeres y Ciencia, CSIC), María Sagrario Millán (vicepresidenta Sociedad Española de Óptica), Pascuala García (presidenta del Grupo Especializado de Mujeres en Física de la RSEF), Ana Jesús López Díaz (Asociación de Mujeres Investigadoras y Tecnólogas AMIT) y María Viñas (OSA Ambassador 2019), representantes de las mayores sociedades científicas de la Óptica y la Fotónica, que nos mostraron una imagen global sobre cómo las sociedades científicas ayudan a visibilizar el papel de la mujer en la ciencia.

El momento emotivo de la jornada llegó con el nombramiento de la Profesora María Luisa Calvo como Socia de Honor de SEDOPTICA, reconocimiento que le entregó la Vicepresidenta de SEDOPTICA, María Millán.

Tras la finalización del Workshop, tuvo lugar la I Asamblea del Área de Mujeres, Óptica y Fotónica de SEDOPTICA, donde se votó la nueva junta directiva y se decidieron los pasos futuros de Área. 
ÓPTICA PURA Y APLICADA

www.sedoptica.es

\begin{tabular}{|l|l|}
\hline I Workshop de Mujeres, Óptica y Fotónica @SEDOPTICA en cifras \\
$-\quad 120$ asistentes \\
- & 15 empresas colaboradoras \\
- & 10 ponencias \\
- & 2 mesas redondas \\
- & 14 bolsas de viaje
\end{tabular}

++ Info: https://areamujersedoptica.wordpress.com/2019/09/17/i-workshop-mujer-optica-y-fotonica/

Un saludo

María Viñas, Verónica González, Clara Benedí Área de Mujer, Óptica y Fotónica de la Sociedad Española de Óptica 